\title{
APPLICATIONS OF FUBINI TYPE THEOREM TO THE TENSOR PRODUCTS OF C*-ALGEBRAS
}

\author{
JUN TOMIYAMA
}

(Received January 8, 1967)

Let $A$ and $B$ be $C^{*}$-algebras and $A \underset{\alpha}{\otimes} B$ their $C^{*}$-tensor product with $\alpha$-norm ([16]). We consider the following linear mapping from $\underset{\alpha}{A \otimes B}$ to $A$ and $B$ defined by

$$
R_{\varphi}\left(\sum_{i=1}^{n} a_{i} \otimes b_{i}\right)=\sum_{i=1}^{n}<a_{i}, \phi>b_{i} \quad\left(\text { resp. } \quad L_{\psi}\left(\sum_{i=1}^{n} a_{i} \otimes b_{i}\right)=\sum_{i=1}^{n}<b_{i}, \psi>a_{i}\right)
$$

for a bounded functional $\boldsymbol{\rho}$ of $A$ (resp. $\psi$ of $B$ ). This mapping satisfies the following relation

$$
\left\langle x, \varphi \otimes \psi>=\left\langle L_{\psi}(x), \varphi>=\left\langle R_{\varphi}(x), \psi>\right.\right.\right.
$$

for every $x \in A \otimes B$.

Now the above relation may be considered, in some sense, as the noncommutative version of Fubini theorem in iterated integrals and it is the purpose of our present discussions to clarify the utility of this result in the tensor products of $C^{*}$-algebras settling all type problems of product algebras (Theorem 2) by using this mapping, and deriving various structure theorems for them, some of which are regarded as the extension of several results in [7], [19].

The above mapping is also useful to more general situations (cf. [10], [15]), since it can be defined in any tensor product of Banach algebras whenever the defining cross-norm is not less than Schatten's $\lambda$-norm ([13]).

Through the discussions $S(A)$ means the set of all states of a $C^{*}$-algebra $A$ and $P(A)$ means the set of all pure states of $A$. The value of a linear functional $\varphi$ on $x$ is always denoted as $\langle x, \varphi\rangle$. Let $A \odot B$ be algebraic tensor product of $A$ and $B$, then the norm $\alpha$ is given by 


$$
\left\|\sum_{i=1}^{n} a_{i} \otimes b_{i}\right\|=\sup \frac{<\left(\sum_{j=1}^{m} x_{j} \otimes y_{j}\right)^{*}\left(\sum_{i=1}^{n} a_{i} \otimes b_{i}\right)^{*}\left(\sum_{i=1}^{n} a_{i} \otimes b_{i}\right)\left(\sum_{j=1}^{m} x_{j} \otimes y_{j}\right), \boldsymbol{\phi} \otimes \psi>^{1 / 2}}{<\left(\sum_{j=1}^{m} x_{j} \otimes y_{j}\right)^{*}\left(\sum_{j=1}^{m} x_{j} \otimes y_{j}\right), \boldsymbol{\phi} \otimes \psi>^{1 / 2}}
$$

where $\varphi$ and $\psi$ run over the set of all states of $A$ and $B$ and $\sum_{j=1}^{m} x_{j} \otimes y_{j}$ runs over $A \odot B$. This norm is also defined as the $C^{*}$-enveloping norm of the product representations $\pi_{1} \otimes \pi_{2}$ of $A \odot B$ where $\pi_{1}$ and $\pi_{2}$ run over irreducible representations of $A$ and $B$, respectively.

1. We state the above cited non-commutative version of Fubini theorem in the following manner.

THEOREM 1. Let $A$ and $B$ be $C^{*}$-algebras, then for any bounded linear functional $\phi$ of $A$ (resp. $\psi$ of $B)$ we can define a linear continuous mapping $R_{\varphi}\left(\right.$ resp. $\left.L_{\psi}\right)$ from $A \otimes B$ onto $B$ (resp. $A$ ) such as

$$
R_{\varphi}\left(\sum_{i=1}^{n} a_{i} \otimes b_{i}\right)=\sum_{i=1}^{n}<a_{i}, \boldsymbol{\rho}>b_{i}\left(\operatorname{resp} . L_{\psi}\left(\sum_{i=1}^{n} a_{i} \otimes b_{i}\right)=\sum_{i=1}^{n}<b_{i}, \psi>a_{i}\right),
$$

satisfying the relation

$$
\left\langle x, \phi \otimes \psi>=\left\langle R_{\varphi}(x), \psi>=\left\langle L_{\psi}(x), \varphi>\right.\right.\right.
$$

for every $x$ in $A \otimes_{\alpha} B$. Mortover the family $\mathfrak{F}_{R}=\left\{R_{\phi} \mid \phi \in S(A)\right\}$ is the total family of positive linear mappings, that is, for any non-zero element $x$ in $A \otimes B$ (not necessarily positive) there exists a state $\phi$ with $R_{\varphi}(x) \neq 0$. The same holds for the family $\mathfrak{F}_{L}=\left\{L_{\psi} \mid \psi \in S(B)\right\}$ and also holds for the families $\left\{R_{\psi} \mid \boldsymbol{\varphi} \in P(A)\right\}$ and $\left\{L_{\psi} \mid \psi \in P(B)\right\}$.

PROOF. We proceed along with $R_{\varphi}$. Since $\alpha$-norm is not less than Schatten's $\lambda$-norm,

$$
\begin{aligned}
& \left\|R_{\varphi}\left(\sum_{i=1}^{n} a_{i} \otimes b_{i}\right)\right\|=\sup _{\|\psi\| \leq 1}\left\|<\sum_{i=1}^{n}<a_{i}, \boldsymbol{\varphi}>b_{i}, \psi>\right\|=\sup _{\|\psi\| \leq 1}\left\|\sum_{i=1}^{n}<a_{i}, \boldsymbol{\varphi}><b_{i}, \psi>\right\| \\
& =\sup _{\mid, \psi i \leq 1}\left|<\sum_{i=1}^{n} a_{i} \otimes b_{i}, \boldsymbol{\varphi} \otimes \psi\right\rangle \mid \leqq \sup _{\|\psi\| \leq 1}\left\|\sum_{i=1}^{n} a_{i} \otimes b_{i}\right\|\|\boldsymbol{\phi}\|\|\psi\|=\|\boldsymbol{\phi}\|\left\|\sum_{i=1}^{n} a_{i} \otimes b_{i}\right\| .
\end{aligned}
$$

so that $R_{\boldsymbol{q}}$ can be extended to the mapping from $A \otimes_{\alpha} B$ onto $B\left(\left\|\mathrm{R}_{\boldsymbol{q}}\right\| \leqq\|\boldsymbol{\rho}\|\right)$. 
The required relation is derived from the identity;

$$
\begin{aligned}
& <\sum_{i=1}^{n} a_{i} \otimes b_{i}, \varphi \otimes \psi>=\sum_{i=1}^{n}\left\langle a_{i}, \varphi><b_{i}, \psi>=<\sum_{i=1}^{n}\left\langle a_{i}, \varphi>b_{i}, \psi>\right.\right. \\
& =<R_{\psi}\left(\sum_{i=1}^{n} a_{i} \otimes b_{i}\right), \psi>=<\sum_{i=1}^{n}\left\langle b_{i}, \psi>a_{i}, \varphi>=<L_{\psi}\left(\sum_{i=1}^{n} a_{i} \otimes b_{i}\right), \boldsymbol{\varphi}\right\rangle .
\end{aligned}
$$

The last half of the theorem is easily seen once we notice that the family of all product functional $\boldsymbol{\otimes} \Psi$ is total on $A \underset{\alpha}{\otimes} B$ and a product functional is a linear combination of product states. The case of pure states is also easily manageable.

We shall show two fundamental properties of these mapping at first.

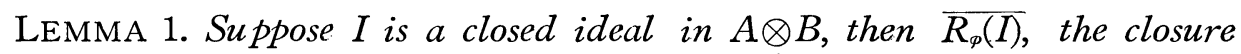
of $R_{\varphi}(I),\left(\right.$ resp. $\left.\overline{L_{\psi}(I)}\right)$ is a closed ideal in $B$ (resp. in $\left.A\right)$.

Proof. Let $R_{\varphi}$ be a fixed map and take an element $x$ in $I$ and an element $b$ in $B$. For an arbitrary positive number $\varepsilon$, choose an element $\sum_{i=1}^{n} x_{i} \otimes y_{i}$ in $A \odot B$ so that $\left\|x-\sum_{i=1}^{n} x_{i} \otimes y_{i}\right\|<\frac{\varepsilon}{\|b\|\|\boldsymbol{\phi}\|}$. By using approximate units in $A$ we can find element $e$ such that

$$
\left\|\sum_{i=1}^{n} x_{i} \otimes b y_{i}-\sum_{i=1}^{n} e x_{i} \otimes b y_{i}\right\|<\frac{\varepsilon}{\|\boldsymbol{\varphi}\|}
$$

Since,

$$
b R_{\varphi}\left(\sum_{i=1}^{n} x_{i} \otimes y_{i}\right)=\sum_{i=1}^{n}<x_{i}, \varphi>b y_{i}=R_{\varphi}\left(\sum_{i=1}^{n} x_{i} \otimes b y_{i}\right),
$$

it can be shown that

$$
\begin{gathered}
\left\|b R_{\varphi}(x)-R_{\varphi}(e \otimes b x)\right\| \leqq\left\|b R_{\varphi}(x)-b R_{\varphi}\left(\sum_{i=1}^{n} x_{i} \otimes y_{i}\right)\right\|+\| R_{\varphi}\left(\sum_{i=}^{n} x_{i} \otimes b y_{i}\right) \\
-R_{\varphi}\left(\sum_{i=1}^{n} e x_{i} \otimes b y_{i}\right)\|+\| R_{\varphi}\left(\sum_{i=1}^{n} e x_{i} \otimes b y_{i}\right)-R_{\varphi}(e \otimes b x) \|<\varepsilon+\varepsilon+\varepsilon=3 \varepsilon
\end{gathered}
$$

and $R(e \otimes b x) \in R_{\varphi}(I)$. Hence $b R_{\varphi}(x) \in \overline{R_{\varphi}(I)}$ and similarly $R_{\varphi}(x) b \in \overline{R_{\varphi}(I)}$. The same argument goes through $L_{\psi}$. 
A composition series $\left\{I_{\lambda}\right\}_{0 \leqq \lambda \leqq \lambda_{0}}$ of a $C^{*}$-algebra $A$ means a well ordered ascending series of closed ideals $I_{\lambda}$ beginning with 0 and ending with $A$, and for any limit ordinal $\lambda, I_{\lambda}$ is the closure of the union of the preceding I's.

LEMMA 2. Let $\left\{I_{\lambda}\right\}_{0 \leqq \lambda \leqq \lambda_{0}}$ be a composition series of a closed ideal $I$ in

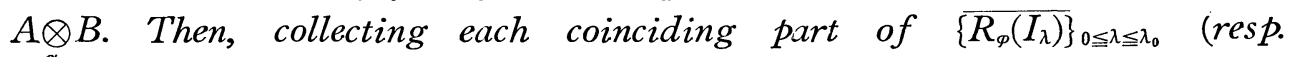

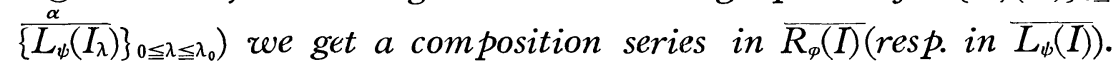

The proof is almost a series of verbal checks, so is left to the reader.

Let $A_{1}$ and $B_{1}$ be $C^{*}$-algebras on a Hilbert space $H_{1}$ and $A_{2}$ and $B_{2}$ be those on $H_{2}$. Then both $C^{*}$-algebras $A_{1} \otimes A_{\alpha}$ and $B_{1} \underset{\alpha}{\otimes} B_{2}$ are considered to be operator algebras on a Hilbert space $H_{1} \otimes H_{2}$. In this case we have

LEMMA 3. If $B_{1} \otimes B_{2}$ is a subalgebra of $A_{1} \otimes A_{2}$, both $B_{1}$ and $B_{2}$ are subalgebras of $A_{1}$ and $A_{2}^{\alpha}$ respectively,

Proof. Suppose that $B_{1}$ is not contained in $A_{1}$ and let $a$ be a non-zero element of $B_{1}$ not belonging to $A_{1}$. We can find a bounded linear functional $\varphi$ on $B\left(H_{1}\right)$, the algebra of all bounded linear operators on $H_{1}$, such that

$$
\langle a, \varphi\rangle \neq 0 \text { and }\langle x, \varphi\rangle=0 \text { for every } x \in A_{1} .
$$

Take a bounded linear functional $\psi$ on $B\left(H_{2}\right)$, the algebra of all bounded linear operators on $H_{2}$, whose restriction to $B_{2}$ does not reduce to zero functional.

The restriction of the product functional $\varphi \otimes \psi$ to $A_{1} \underset{\alpha}{\otimes A_{2}}$ is zero, whereas the restriction to $B_{1} \otimes_{\alpha} B_{2}$ does not reduce to zero, a contradiction. Hence $A_{1} \supset B_{1}$ and similorly ${ }^{\alpha} A_{2} \supset B_{2}$.

In the following, we denote by $\pi_{\varphi}$ the canonical representation associated with a state $\varphi$ of a $C^{*}$-algebra. $H_{\pi}$ means the representation space of a representation $\pi$. We also denote by $C(H)$ the $C^{*}$-algebra of all compact operators on a Hilbert space $H$. An irreducible representation $\pi$ of a $C^{*}$. algebra $A$ is called normal if $\pi(A)$ contains a (non-zero) compact operator of $H_{\pi}$ (hence necessarily $\pi(A) \supset C(H)$ by the result of [5]).

An immediate corollary of Lemma 3 is the following result concerning normal representations without the assumption of separability.

COROLLARY. Let $\pi_{1}$ and $\pi_{2}$ be irreducible representations of $C *$-algebras $A$ and $B$. The product representation $\pi_{1} \otimes \pi_{2}$ of $A \otimes_{\alpha} B$ is normal if and only if both $\pi_{1}$ and $\pi_{2}$ are normal.

Now we shall decide all types of tensor products of $C^{*}$-algebras. Though 
proofs are separated we shall state all of them together for completeness in the next

THEOREM 2. Let $A$ and $B$ be $C *$-algebras. Then,

(a) $A \otimes{ }_{\alpha} B$ is a $C^{*}$-algebras with continuous trace if and only if both $A$ and $B$ are $C^{*}$-algebras with continuous trace,

(b) $A \otimes B$ is a generalized $C *$-algebra with continuous trace (abbreviated by GTC-algebra) if and only if both $A$ and $B$ are GTC-algebras,

(c) $A \otimes_{\alpha} B$ is a CCR algebra if and only if both $A$ and $B$ are CCR algebras,

(d) $A \underset{\alpha}{\otimes} B$ is a GCR algebra if and only if both $A$ and $B$ are GCR algebras,

(e) $A \otimes B$ is an NGCR algebra if and only if either $A$ or $B$ is an NGCR algebra.

A part of these results is found in Wulfsohn [18], [19] and Guichardet ([7] some of them with the assumption of separability). It is also to be noticed that using Sakai's recent result [12] we can prove the assertion (e) along with the same line as Guichardet [7] used the results of Glimm [6] in separable case, but our proof of (e) is more direct and uses only elementary properties avoiding to use the results of Glimm's difficult constructions.

Indeed the assertion (e) is an easy consequence of the following result which seems to have an interest of its own.

THEOREM 3. Let I be a CCR ideal in $A \underset{\alpha}{\otimes} B$ and $\boldsymbol{\phi}$ be a pure state of $A$ (resp. B). Then $\overline{R_{\varphi}(I)}$ (resp. $\overline{\left.L_{\varphi}(I)\right)}$ is a CCR ideal in $B$ (resp. in $\left.A\right)$. If $I$ is a GCR ideal in $A \underset{\alpha}{\otimes B}, \overline{R_{\varphi}(I)}\left(\right.$ resp. $\left.\overline{L_{\psi}(I)}\right)$ is also GCR ideal in $B$ (resp. in $\left.A\right)$.

Proof. We may assume that $R_{\varphi}(I) \neq 0$. Write $\phi={ }^{t} \pi_{\varphi}\left(\omega_{\xi}\right)$ where $\omega_{\xi}$ is a vector state of $\pi_{\varphi}(A)$ by a vector $\xi(\|\xi\|=1)$ in $H_{\pi_{\varphi}}$. Let $\psi$ be a pure state of $B$ such that $\pi_{\psi} \mid \overline{R_{\varphi}(I)}$, the restriction of $\pi_{\psi}$ to $\overline{R_{\varphi}(I)}$, is a non-zero representation of a $C^{*}$-algebra $\overline{R_{\varphi}(I)}$.

For any element $x$ in $A \underset{\alpha}{\otimes} B$ and a bounded linear functional $\phi$ of $\pi_{\psi}(B)$ we have by Theorem 1

$$
\begin{aligned}
& <\pi_{\psi}\left(R_{\varphi}(x)\right), \phi>=<R_{\varphi}(x),{ }^{t} \pi_{\psi}(\phi)>=<x, \varphi \otimes{ }^{t} \pi_{\psi}(\phi)> \\
& =<x,{ }^{t} \pi_{\varphi}\left(\omega_{\xi}\right) \otimes{ }^{t} \pi_{\psi}(\phi)>=<x,{ }^{t}\left(\pi_{\varphi} \otimes \pi_{\psi}\right)\left(\omega_{\xi} \otimes \phi\right)>=<\pi_{\varphi}^{-*} \otimes \pi_{\psi}(x), \omega_{\xi} \otimes \phi>\cdots\left(^{*}\right)
\end{aligned}
$$


Since $\pi_{\psi} \mid R_{\varphi}(I) \neq 0$, the above relation implies that $\pi_{\varphi} \otimes \pi_{\psi} \mid I \neq 0$, so that $\pi_{\varphi} \otimes \pi_{\psi}$ becomes an irreducible representation of $I$ and as $I$ is a $C C R$ ideal,

$$
\pi_{\varphi} \otimes \pi_{\psi}(I)=C\left(H_{\pi_{\varphi}} \otimes H_{\pi_{\varphi}}\right)=C\left(H_{\pi_{\varphi}}\right) \underset{\alpha}{\otimes} C\left(H_{\pi_{\psi}}\right) .
$$

Hence $\pi_{\psi}(B) \supset C\left(H_{\pi_{\phi}}\right)$ by Lemma 3 . Therefore the equality (*) also implies that the polar of $\pi_{\psi}\left(R_{\varphi}(I)\right)$ and that of $C\left(H_{\pi_{\varphi}}\right)$ in the conjugate space of $\pi_{\psi}(B)$ coincide each other, which shows $\pi_{\psi} \overline{\left(R_{\varphi}(I)\right)}=\pi_{\psi} \overline{\left(R_{\varphi}(I)\right)}=C\left(H_{\pi q}\right)$. As every irreducible representations of $\overline{R_{\varphi}(I)}$ arise in this way, $\overline{R_{\varphi}(I)}$ must be $C C R$ ideal in $B$.

Next, let $I$ be a $G C R$ ideal in $A \otimes B$ and $\left\{I_{\lambda}\right\}_{0 \leqq \Lambda \leqq \lambda_{0}}$ a canonical composition series for $I$. Denote by $\left\{J_{\Lambda}\right\}_{0 \leqq 1 \leqq \Lambda_{0}}$ the derived composition series of $\overline{R_{\varphi}(I)}$ by $R_{\varphi}\left(\right.$ cf. Lemma 2) and let $\psi$ be a pure state of $B$ such that $\pi_{\psi} \mid J_{\Lambda}=0$ and $\pi_{\psi} \mid J_{\Lambda+1} \neq 0$ for some index $\Lambda$. The representation $\pi_{\psi}$ can be considered naturally as an irreducible representation of a $C^{*}$-algebra $J_{A+1} / J_{\Lambda}$. Consider the corresponding family of the ideal $I$ 's with $\overline{R_{q}\left(I_{\lambda}\right)}=J_{A+1}$. There exists the smallest ideal $I_{\lambda}$ among them and in this case $\lambda$ is not a limit ordinal. Thus $\lambda=\lambda_{1}+1$ and clearly $\overline{R_{\varphi}\left(I_{\lambda_{1}}\right)}=J_{\Lambda}$.

Now for a vector state $\omega_{\eta}$ in $H_{\pi_{\varphi}}$ the equality (*) turns out to be

$$
<\pi_{\psi}\left(R_{\varphi}(x)\right), \omega_{\eta}>=<\pi_{\varphi} \otimes \pi_{\psi}(x), \omega_{\xi} \otimes \omega_{\eta}>=<\pi_{\varphi} \otimes \pi_{\psi}(x), \omega_{\xi \searrow \eta}>.
$$

Hence $\pi_{\psi} \mid J_{A}=0$ implies that the restriction of a vector state $\omega_{\xi \otimes \eta}$ to $\pi_{\varphi} \otimes \pi_{\psi}\left(I_{\lambda_{1}}\right)$ is zero, which implies $\pi_{\varphi} \otimes \pi_{\psi} \mid I_{\lambda_{1}}=0$. On the other hand, $\pi_{\varphi} \otimes \pi_{\psi} \mid I \neq 0$ because $\pi_{\psi} \mid R_{\varphi}\left(I_{\lambda_{1}+1}\right) \neq 0$. Therefore $\pi_{\varphi} \otimes \pi_{\psi}$ can be considered to be an irreducible representation of $I_{\lambda_{1}+1} / I_{\lambda_{1}}$ which is a $C C R$ algebra by assumption. Then the same argument as in the proof of the first half part of the theorem shows that

$$
\pi_{\psi}\left(J_{\Lambda+1} / J_{\Lambda}\right)=\pi_{\psi}\left(J_{\Lambda+1}\right)=\pi_{\varphi}\left(\overline{R_{\varphi}\left(I_{\lambda_{1}+1}\right)}\right)=C\left(H_{\pi_{\psi}}\right)
$$

and since each irreducible representation of $J_{A+1} / J_{A}$ arises in this way $J_{A+1} / J_{A}$ is a $C C R$ algebra. This completes the proof. Similar arguments show that the results also hold for $L_{\varphi}$.

An immediate consequence of Theorem 3 is the only if parts of the assertions (c) and (d). A direct proof of the if parts of (c) and (d) are found in [18] and [19]. The proof of the assertion (e) goes as follows; if either $A$ or $B$ is an $N G C R$ algebra there exist no non-zero $G C R$ ideals in $A \otimes B$ by Theorem 1 and 3 , hence $A \underset{\alpha}{\otimes B}$ is an $N G C R$ algebra. The only if part ${ }^{\alpha}$ of the assertion (e) is an easy consequnce of the if part of (d). Direct proofs of the 
if parts of the assertions (a) and (b) are due to Wulfsohn [19]. But, before going into our final discussions we shall quote here the definitions of a $C$. algebra with continuous trace and a GTC algebra following Dixmier [4].

Let $\operatorname{Tr} \pi(a)$ be the trace of the operator $\pi(a)$ for an irreducible representation $\pi$ of a $C^{*}$-algebra $A$ and a positive element $a$ in $A$. Since $\operatorname{Tr} \pi(a)$ only depends on the unitary equivalent class of $\pi, \operatorname{Tr} \pi(a)$ is considered a function on $\hat{A}$, the dual space of $A$. In the following we shall identify an irreducible representation $\pi$ with $\widehat{\pi}$, the unitary equivalence class to which $\pi$ belongs. Let $\mathfrak{p}$ be the set of all positive elements $a$ in $A$ such that $\operatorname{Tr} \pi(a)$ is a finite continuous function on $\hat{A}$, then there exists a self-adjoint two-sided ideal $\mathfrak{m}(A)$ in $A$ whose positive part coincides with $\mathfrak{p}$. Put $J(A)=\overline{\mathfrak{m}(A)}$, the closure of $\mathfrak{m}(A) . A$ is called a $C *$-algebra with continuous trace if $J(A)=A$. On the other hand, generally we can find a composition series $\left\{I_{\lambda}\right\}_{0 \leq \lambda \leq \lambda_{0}}$ of a closed ideal $I_{\lambda_{0}}$ in $\mathrm{A}$ such as $J\left(A / I_{\lambda_{0}}\right)=0$ and $J\left(A / I_{\lambda}\right)=I_{\lambda_{1}} / I_{\lambda}$. If $I_{\lambda_{0}}=A$, we call $A$ a GTC algebra and $\left\{I_{\lambda}\right\}$ the canonical composition series of $A$. We shall use the following characterization of GTC algebras by [4; proposition 12 and 13], that is, a $C^{*}$-algebra $A$ is a GTC algebra if and only if there exists a well ordered ascending series $\left\{U_{\lambda}\right\}$ of open sets in $\widehat{A, \text { beginning with null set and }}$ ending with $\hat{A}$, and such that if $\lambda$ is a limit ordinal $U_{\lambda}$ is the union of the preceding $U$ 's and each point in $U_{\lambda+1}-U_{\lambda}$ admits a fundamental system of closed neighbrohoods in $\widehat{A}-U_{\lambda}$.

LEMMA 4. Let $\phi$ be a pure state of $A$ (resp. B). Then $R_{\varphi}(J(A \otimes B)) \subset J(B)$ (resp. $\left.L_{\varphi}(J(A \underset{\alpha}{\otimes} B)) \subset J(A)\right)$.

ProOF. It is sufficient to show that $R_{\varphi}\left(\mathfrak{m}(A \otimes B)^{+}\right) \subset \mathfrak{m}(B)^{+}$where the sign " + " indicates positive parts of algebras. We choose a complete orthonormal basis $\left\{\xi_{i}\right\}$ in $H_{\pi_{\varphi}}$ so that $\phi={ }^{t} \pi_{\varphi}\left(\omega_{\xi_{0}}\right)$. Let $\psi$ be a pure state of $B$ and $\left\{\boldsymbol{\eta}_{k}\right\}$ be a complete orthonormal basis in $H_{\pi_{\psi^{*}}}$. Note that $\left\{\xi_{i} \otimes \eta_{k}\right\}$ is a complete orthonormal basis in $H_{\pi_{\varphi}} \otimes H_{\pi_{\psi^{*}}}$. For an arbitrary element $a$ in $\mathfrak{m}(A \underset{\alpha}{\otimes} B)^{+}$,

$$
\operatorname{Tr}\left(\pi_{\varphi} \otimes \pi_{\psi}(a)\right)=\sum_{i, k}\left(\pi_{\varphi} \otimes \pi_{\psi}(a) \xi_{i} \otimes \eta_{k}, \xi_{i} \otimes \eta_{k}\right)<\infty
$$

by assumptions. Therefore,

$$
\begin{aligned}
\operatorname{Tr}\left(\pi_{\varphi} \otimes \pi_{\psi}(a)\right) & =\sum_{i} \sum_{k}\left(\pi_{\varphi} \otimes \pi_{\psi}(a) \xi_{i} \otimes \eta_{k}, \xi_{i} \otimes \eta_{k}\right) \\
& =\sum_{i} \sum_{k}<\pi_{\varphi} \otimes \pi_{\psi}(a), \omega_{\xi_{i} \otimes \eta_{k}}>=\sum_{i} \sum_{k}<\pi_{\varphi} \otimes \pi_{\psi}(a), \omega_{\xi_{i}} \otimes \omega_{\eta_{k}}>
\end{aligned}
$$




$$
\begin{aligned}
& =\sum_{i} \sum_{k}<a,{ }^{t} \pi_{\varphi}\left(\omega_{\xi_{t}}\right) \otimes{ }^{t} \pi_{\psi}\left(\omega_{\eta_{k}}\right)>=\sum_{i} \sum_{k}<R_{\pi_{\pi_{\varphi}}\left(\omega_{\xi_{t}}\right)}(a),{ }^{t} \pi_{\psi}\left(\omega_{\eta_{k}}\right)> \\
& =\sum_{i} \sum_{k}\left(\pi_{\psi}\left(R_{\left.\pi_{\pi_{\varphi}\left(\omega_{\xi_{i}}\right.}\right)}(a)\right) \eta_{k}, \eta_{k}\right)=\sum_{i} \operatorname{Tr}\left(\pi _ { \psi } \left(R_{\left.\left.\pi_{\pi_{\varphi}\left(\omega_{\xi_{i}}\right.}(a)\right)\right) .}\right.\right.
\end{aligned}
$$

Hence,

$$
\operatorname{Tr}\left(\pi_{\psi}\left(R_{\varphi}(a)\right)\right)=\operatorname{Tr}\left(\pi_{\varphi} \otimes \pi_{\psi}(a)\right)-\sum_{i \neq 0} \operatorname{Tr}\left(\pi_{\psi}\left(R_{t_{\pi_{\varphi}\left(\omega_{\xi_{i}}\right)}}(a)\right)\right)
$$

Now the first term of right hand is continuous in $\pi_{\varphi} \otimes \pi_{\psi} \in \widehat{A \otimes B}$, the dual space of $A \otimes B$, hence continuous in $\pi_{\psi}$ fixing $\pi_{\varphi}$, while the second term is the sum of positive lower semi-continuous functions (cf. [3; Proposition 5]) with variable $\pi_{\psi}$ hence itself lower semi-continuous. Thus $\operatorname{Tr}\left(\pi_{\psi}\left(R_{\varphi}(a)\right)\right)$ is a finite upper semi-continuous function on $\widehat{B}$ and as $\operatorname{Tr}\left(\pi_{\psi}\left(R_{\varphi}(a)\right)\right)$ is always lower semi-continuous in $\widehat{B}$ ([3]) this concludes the proof. The proof for $L_{\varphi}$ is almost the same.

Since $R_{\varphi}$ and $L_{\psi}$ are onto mappings Lemma 4 contains the only if part of the assertion (a).

At last, suppose that $A \otimes B$ is a $G T C$-algebra. Take a pure state $\phi$ of $A$ and let $\left\{J_{A}\right\}_{0 \leqq 1 \leqq A_{0}}$ be a composition series of $B$ derived by Lemma 2 from the canonical composition series $\left\{I_{\lambda}\right\}_{0 \leqq \lambda \leqq \lambda_{0}}$ of $A \otimes B$. The duals of all $J^{\prime} \mathrm{s}$ form a well ordered ascending series of open sets in ${ }^{\alpha} \widehat{B}$, beginning with null set and ending with $\widehat{B}$, and such that if $\Lambda$ is a limit ordinal $\widehat{J}_{A}$ is the union of the preceding $\widehat{J}$ 's. Take an element $\pi_{0}$ in $\widehat{J}_{A+1}-\widehat{J}_{A}$. The arguments in the proof of the last half part of Theorem 3 show that there exists an index $\lambda$ satisfying $\overline{R_{\varphi}\left(I_{\lambda+1}\right)}=J_{\Lambda+1}$ and $\overline{R_{\varphi}\left(I_{\lambda}\right)}=J_{\Lambda}$ and that $\pi_{\varphi} \otimes \pi_{0}$ belongs to $\widehat{I}_{\lambda+1}-\widehat{I}_{\lambda}$.

Now a $G T C$-algebra is a $G C R$ algebra, hence a $C$ *-algebra of type I. Thus by (d) and [18; Theorem 4] we can identify the topological space $\widehat{A \otimes B} \underset{\alpha}{\otimes}$ with $\widehat{A} \times \widehat{B}$ (the identification goes through $\pi_{1} \otimes \pi_{2}=\left(\pi_{1}, \pi_{2}\right)$ ). Let $U$ be an arbitrary neighborhood of $\pi_{0}$ in $\widehat{B}$. Denoting by $V$ an open neighborhood of $\pi_{\phi}$ in $\widehat{A}$ we get a neighborhood $V \times U$ of $\pi_{\varphi} \otimes \pi_{0}$. Hence there exists an open neighborhood $W(\subset V \times U)$ of $\pi_{\varphi} \otimes \pi_{0}$ such that

$$
\left.\overline{W \cap\left(\widehat{A} \times \widehat{B}-\widehat{I_{\lambda}}\right.}\right) \subset V \times U \cap\left(\widehat{A} \times \widehat{B}-\widehat{I_{\lambda}}\right)
$$

by the above cited result in [4]. For this neighborhood $W$ we can find open neighborhoods $V_{1}$ and $U_{1}$ of $\pi_{\varphi}$ and $\pi_{0}$, satisfying $V_{1} \times U \subset W$. Then one easily sees that 


$$
\left.\overline{U_{1} \cap\left(\widehat{B}-\widehat{J}_{A}\right.}\right) \subset U \cap\left(\widehat{B}-\widehat{J}_{\Lambda}\right)
$$

that is, $\pi_{0}$ admits in $\widehat{B}-\widehat{J}_{A}$ a fundamental system of closed neighborhoods. Therefore $B$ is a $G T C$-algebra and similarly $A$ is a $G T C$-algebra, too. Thus all proofs of Theorem 2 are completed.

2. Here we consider at first the types of general tensor products $A \otimes B$ of $C *$-algebras $A$ and $B$ by a compatible norm $\beta$. $A$ cross-norm $\beta$ in $A \bigodot^{\beta} B$ is called a compatible norm if the completion of the normed *-algebra $A \odot B$ by $\beta$-norm becomes a $C *$-algebra. That is, $\beta$ is the norm satisfying the following conditions

$$
\begin{aligned}
& \left\|\left(\sum_{i=1}^{n} a_{i} \otimes b_{i}\right)\left(\sum_{j=1}^{m} c_{j} \otimes d_{j}\right)\right\| \leqq\left\|\sum_{i=1}^{n} a_{i} \otimes b_{i}\right\|\left\|\sum_{j=1}^{m} c_{j} \otimes d_{j}\right\|, \\
& \left\|\left(\sum_{i=1}^{n} a_{i}^{*} \otimes b_{i}^{*}\right)\left(\sum_{i=1}^{n} a_{i} \otimes b_{i}\right)\right\|=\left\|\sum_{i=1}^{n} a_{i} \otimes b_{i}\right\|^{2}, \\
& \text { and }\|a \otimes b\|=\|a\|\|b\| .
\end{aligned}
$$

Among these norms 'Turumaru's $\alpha$-norm is the smallest one (Takesaki [14; Theorem 2]). Hence a product functional $\varphi \otimes \psi$ is always continuous on $A \otimes_{\beta} B$. On the other hand, there exists the largest compatiale norm $\nu$ as shown in Guichardet [8]. $A \otimes B$ is nothing but the enveloping $C^{*}$-algebra of an involutive Banach algebra $A \otimes B$, tensor product by $\gamma$-norm in the sense of Schatten [13] (cf. [11]). Following ${ }^{\gamma}[14]$, we say that a $C^{*}$-algebra $A$ bas the property $(T)$ if $\alpha$-norm is the unique compatible norm in $A \odot B$ for any $C^{*}$-algebra $B$. $A G C R$ algebra has the property $(T)$ by [14; Theorem 3], hence there are no problems for compatible norms in the corresponding if parts of the assertions (a), (b), (c) and (d) in Theorem 2. On the other hand, suppose that $A \otimes B$ is a $G C R$ algebra. Since $\beta \geqq \alpha, A \otimes B$ becomes a homomorphic image of a $G \stackrel{\beta}{C} R$ algebra, $A \otimes_{\beta} B$, hence itself a $G C R$ algebra. Therefore both $A$ and $B$ are $G C R$ algebras by the assertion (d) of Theorem 2 and a posteriori $\beta=\alpha$. As all classes of $C^{*}$-algebras in the assertions (a), (b) and (c) are GCR algebras, the above result shows that there are no distinguished points in the corresponding only if parts in (a), (b) and (c) for a compatible norm from those in Theorem 2. As to the assertion (e) the only if part holds for any compatible norm $\beta$. In fact, suppose that $A \otimes B$ is an $N G C R$ algebra and that both $A$ and $B$ have non-zero $G C R$ ideals $I^{\beta}$ and $J$ respectively. Then the closure of $I \odot J$ in $A \underset{\beta}{\otimes} B$ is a non-zero $G C R$ ideal by the above consideration, a contradiction. Hence either $A$ or $B$ 
is an NGCR algebra. However the author does not know whether or not $A \otimes B$ is an $N G C R$ algebra when $A$ or $B$ is an $N G C R$ algebra.

Next, let $K_{1}, K_{2}$ and $K$ be the largest $G C R$ ideals in $A, B$ and $A \otimes B$ respectively. It is plausible that generally $K_{1} \underset{\alpha}{\otimes} K_{2}=K$, which will clarify the reason of the assertions (d) and (e) in Theorem 2. Unfortunately, we get only the following partial result.

Theorem 4. Let $A$ and $B$ be $C *$-algebras. If either $A$ or $B$ has the p.operty (T), then $K_{1} \underset{\alpha}{\otimes} K_{2}=K$ in $A \underset{\alpha}{\otimes} B$.

Proof. Assume that $A$ has the property (T), i. e. $\alpha=\nu$ in any $C *$-tensor product $A \otimes B$. It is shown in [8] that the kernel of the canonical homomorphism $A \otimes B$ to $A \otimes B / K_{2}$ induced by the quotient homomorphism $B \rightarrow B / K_{2}$ is $A \otimes K_{2}$. If $K_{2} \neq B, \stackrel{\nu}{A} \otimes B / K_{2}=A \otimes B / K_{2}$ is an $N G C R$ algebra by the assertion (e) in "Theorem 2 and ${ }^{\nu} A \otimes K_{2} \supset K$. The case $K_{2}=B$ also implies that $A \otimes K_{2}$ $=A \otimes B \supset K$. Next, consider the homomorphism $A \otimes K_{2} \rightarrow A / K_{1} \otimes K_{2}$ induced by the quotient homomorphism $A \rightarrow A / K_{1}$. Then similar arguments as above show that its kernel $K_{1} \otimes K_{2}$ (cf. [8]) contains $K$. As a $G C R$ algebra has the property (T), $K_{1} \otimes K_{2}$ coincides with $K_{1} \otimes K_{2}$ and $K_{1} \otimes K_{2} \supset K$, while the converse inclusion is seen from the assertion (d) in Theorem 2.

As for $J(A), J(B)$ and $J(A \otimes B)$ it can be shown that if $K_{1} \otimes K_{2}=K$ holds in $A \otimes_{\alpha} B$ we have $J(A) \underset{\alpha}{\otimes} J(B) \stackrel{\alpha}{=} J(A \underset{\alpha}{\otimes} B)$. We omit the proof.

3. Let $\pi$ be an irreducible representation of $A \otimes B$ on $H_{\pi}$. As it is known, $\pi$ induces canonically factor representations $\pi_{1}$ and $\pi_{2}$ of $A$ and $B$ on $H_{\pi}$ such that $\pi(a \otimes b)=\pi_{1}(a) \pi_{2}(b)=\pi_{2}(b) \pi_{1}(a)$ (cf.[7]) and there exists a natural isomorphism between the algebraic generation by $\pi_{1}(A)$ and $\pi_{2}(B)$ and $\pi_{1}(A) \bigodot \pi_{2}(B)$ (cf. for example [1; Chap. $1 \S 2$, Exercise 6]). Hence transposing the norm in the algebraic generation by $\pi_{1}(A)$ and $\pi_{2}(B)$ to $\pi_{1}(A) \odot \pi_{2}(B)$ we get the tensor product $\pi_{1}(A) \bigotimes_{\beta} \pi_{2}(B)$ which is necessarily isomorphic to $\pi(A \otimes B)$. These represenattions $\pi_{1}$ and $\pi_{2}$ are called restrictons of $\pi$ to $A$ and $B$. Now let $I$ be a proper closed ideal in $A \otimes_{\alpha} B$ and $\pi$ be an irreducible representation of $A \otimes B$ vanishing on $I$. Denote by ${ }^{\alpha} \phi$ and $\psi$ pure states of $\pi_{1}(A)$ and $\pi_{2}(B)$ and by $\theta_{\pi}$ the canonical isomorphism between $\pi^{\prime}(A \underset{\alpha}{\otimes} B)$ to $\pi_{1}(A) \bigotimes_{\beta} \pi_{2}(B)$, then an easy calculation shows that ${ }^{t} \pi^{t}{ }^{t} \theta_{\pi}(\boldsymbol{\phi} \otimes \psi)={ }^{t} \pi_{1}(\phi){ }^{\alpha} \otimes{ }^{t} \pi_{2}(\psi)$ and ${ }^{\beta}$ we get the following result.

"For each proper closed ideal I in $A \otimes B$, we can find a pure state ${ }^{t} \pi_{1}(\phi)$ $\otimes^{t} \pi_{2}(\psi)$ vanishing on $I^{\prime \prime}$. 
Combinning this with Theorem 1 we can show that the $\alpha$-tensor product of simple $C^{*}$-algebras is also simple. In fact, let $A$ and $B$ be both simple $C^{*}$ algebras and $I$ be a proper closed ideal in $A \otimes B$. There exists a pure state $\phi_{0} \otimes \psi_{0}$ vanishing on $I$, and $\left\langle I, \varphi_{0} \otimes \psi_{0}\right\rangle=\left\langle\stackrel{\alpha}{R}_{\phi_{0}}(I), \psi_{0}\right\rangle=0$. Hence $\overline{R_{\varphi_{0}}(I)}$ is a proper closed ideal in $B$ by Lemma 1 , and $R_{\varphi_{0}}(I)=0$. Therefore for an arbitrary pure state $\psi$ of $B$,

$$
<L_{\psi}(I), \varphi_{0}>=<I, \varphi_{0} \otimes \psi>=<R_{\varphi_{0}}(I), \psi>=0 .
$$

As $\mathrm{A}$ is also simple, this means $\overline{L_{\psi}(I)}=0$ i.e. $L_{\psi}(I)=0$. Hence $I=0$ by Theorem 1.

This fact is proved at first in [14].

Now the question naturally arises; when does the family of all (product) pure states $\varphi \otimes \psi$ of $A \underset{\alpha}{\otimes} B$ separates closed ideals in $\underset{\alpha}{A} B$ ? This question means that to what extent a closed ideal in $A \otimes B$ is determined by its components in $A$ and $B$ and that, in other words, to what extent the quotient algebras of $A \otimes B$ are compatible with Fubini type theorem described in Theorem 1. So we set the following definition.

DEFInItion. $A \otimes B$ is called to satisfy the condition $(F)$ if the family $\left\{\boldsymbol{\rho} \otimes \psi \mid \boldsymbol{\psi} \in P(A)\right.$ and $\left.\psi_{\boldsymbol{\alpha}} \in P(B)\right\}$ separates all closed ideals in $A_{\alpha} B$.

Before going into the structure theorem of the algebra satisfying condition $(F)$ we note that a similar argument as in the first part of this section shows that there exists a canonical isomorphism $\theta_{\pi}$ between the image $\pi(A \otimes B)$ of a factor representation $\pi$ of $A \underset{\alpha}{\otimes} B$ and $\pi_{1}(A) \underset{\beta}{\otimes} \pi_{2}(B)$ such as

$$
\theta_{\pi} \circ \pi\left(\sum_{i=1}^{n} a_{i} \otimes b_{i}\right)=\theta_{\pi}\left(\sum_{i=1}^{n} \pi_{1}\left(a_{i}\right) \pi_{2}\left(b_{i}\right)\right)=\sum_{i=1}^{n} \pi_{1}\left(a_{i}\right) \otimes \pi_{2}\left(b_{i}\right)
$$

where $\pi_{1}$ and $\pi_{2}$ are restrictions of $\pi$ to $A$ and $B$.

THEOREM 5. The following statements for $A \underset{\alpha}{\otimes} B$ are equivalent;

1. $A \otimes B$ satisfies the condition $(F)$,

2. for each closed ideal $I$ in $A$ and $J$ in $B$, the kernel of the homomorphism $A \otimes B \rightarrow A / I \otimes B / J$ canonically induced by the quotient homomorphisms $A \rightarrow A / I$ and $B \rightarrow B / J$ is given by $I \underset{\alpha}{\otimes} B+A \underset{\alpha}{\otimes} J$.

3. for any factor representation $\pi$ of $A \underset{\alpha}{\otimes} B, \pi(A \underset{\alpha}{\otimes} B)$ is canonically isomorphic to the $\alpha$-tensor product $\pi_{1}(A) \underset{\alpha}{\otimes \pi_{2}}(B) \stackrel{\alpha}{\text { where }} \pi_{1}^{\alpha}$ and $\pi_{2}$ are restrictions of $\pi$ to $A$ and $B$. 
If either $A$ or $B$ is of type I, $A \otimes B$ satisfies the condition $(F)$ as seen from the condition 3 and [14; Theorem 3]. Hence Theorem 5 explains the back of Theorem 1 in [19]. Moreover, since the homomorphic images of the $C^{*}$ algebra which is an inductive limit of $C^{*}$-subalgebras of type $\mathrm{I}$ in the sense of Takeda [20] are also algebras of the same type, we can see by Theorem 5 in [14] and the condition 3 that $A \otimes B$ satisfies the condition $(F)$ if $A$ or $B$ is an inductive limit of $C^{*}$-subalgebras of type $\mathrm{I}$.

Proof of Theorem 5. The implication $1 \rightarrow 2$. Let $I$ and $J$ be closed ideals in $A$ and $B$ and $\theta_{1}, \theta_{2}$ be the quotient homomorphism $A \rightarrow A / I$ and $B \rightarrow B / J$. Denote by $\theta_{1} \otimes \theta_{2}$ the canonical homomorphism $A \underset{\alpha}{\otimes} B \rightarrow A / I \otimes \otimes_{\alpha} B / J$ induced by them. Clearly the kernel of $\theta_{1} \otimes \theta_{2},\left(\theta_{1} \otimes \theta_{2}\right)^{-1}(0)$, contains the ideal $I \otimes B+A \otimes J$. Suppose that $\phi \otimes \psi \mid I \otimes B+A \otimes J=0$ where $\phi \in P(A)$ and $\psi \in P(B)$. We have ${ }^{\alpha} \phi \mid I=0$ and $\psi \mid J=0$, so that $\varphi^{\alpha}$ and $\psi$ induce pure states $\phi^{\prime}$ and $\psi^{\prime}$ on $A / I$ and $B / J$. Then for an arbitrary element $x$ in $\left(\theta_{1} \otimes \theta_{2}\right)^{-1}(0)$,

$$
<x, \varphi \otimes \psi>=<x,{ }^{t} \theta_{1}\left(\phi^{\prime}\right) \otimes{ }^{t} \theta_{2}\left(\psi^{\prime}\right)>=<x,{ }^{t}\left(\theta_{1} \otimes \theta_{2}\right)\left(\phi^{\prime} \otimes \psi^{\prime}\right)>=0 .
$$

Therefore the condition 1 implies that $\left(\theta_{1} \otimes \theta_{2}\right)^{-1}(0)=I \otimes B+A \otimes J$.

The implication $2 \rightarrow 3$. Let $\pi$ be a factor representation of $A_{\alpha}^{\alpha} B$ and $\pi_{1}$, $\pi_{2}$ its restrictions to $A$ and $B$. As we said above, there exists a compatible norm $\beta$ in $\pi_{1}(A) \odot \pi_{2}(B)$ such that $\pi(A \underset{\alpha}{\otimes} B)$ is isomorphic to $\pi_{1}(A) \underset{\beta}{\otimes} \pi_{2}(B)$ by $\theta_{\pi}$. Let $\rho$ be the canonical homomorphism from $\pi_{1}(A) \underset{\beta}{\otimes} \pi_{2}(B)$ to $\pi_{1}^{\beta}(A) \underset{\alpha}{\otimes} \pi_{2}(B)$. The composed homomorphism $\rho \circ \theta_{\pi} \circ \pi$ is nothing but the product homomorph $\stackrel{\beta}{\mathrm{\beta} m}$ from $A \otimes_{\alpha} B$ to $\pi_{1}(A) \otimes_{\alpha} \pi_{2}(B)$ induced by $\pi_{1}$ and $\pi_{2}$. As the latter is a composition of the homomorphism ${ }^{\alpha} A \otimes B \rightarrow A / \pi_{1}^{-1}(0) \underset{\alpha}{\otimes} B / \pi_{2}^{-1}(0)$ and the isomorphism between $A / \pi_{1}^{-1}(0) \otimes_{\alpha} B / \pi_{2}^{-1}(0)$ and $\pi_{1}^{\alpha}(A) \underset{\alpha}{\otimes} \pi_{2}(B)$, the kernel of $\rho \circ \theta_{\pi} \circ \pi$ is $\pi_{1}^{-1}(0) \otimes_{\alpha} B+A \otimes \pi_{2}^{-1}(0)$ by the assumption 2 . On the other hand,

$$
\pi_{1}^{-1}(0) \underset{\alpha}{\otimes} B+A \underset{\alpha}{\otimes} \pi_{2}^{-1}(0) \subset \pi^{-1}(0) \subset \text { the kernel of } \rho \circ \theta_{\pi} \circ \pi .
$$

Hence $\pi^{1}(0)=\pi_{1}^{-1}(0) \underset{\alpha}{\otimes} B+A \otimes_{\alpha} \pi_{2}^{-1}(0)$, and $\rho$ is an isomorphism, that is, $\beta=\alpha$.

The implication $3 \rightarrow 1$. Let $I$ and $J$ be distinct closed ideals in $A \underset{\alpha}{\otimes} B$. We may assume that $I$ is not contained in $J$. Take an irreducible representation $\pi$ of $A \otimes B$ such that $\pi(I) \neq 0$ and $\pi(J)=0$ and let $\pi_{1}$ and $\pi_{2}$ be its restrictions. We have $\theta_{\pi} \circ \pi(I) \neq 0$ in $\pi_{1}(A) \underset{\alpha}{\otimes} \pi_{2}(B)$ and we can find pure states $\varphi$ and $\psi$ of $\pi_{1}(A)$ and $\pi_{2}(B)$ such as $\phi \otimes \psi \mid \theta_{\pi} \circ \pi(I) \neq 0$. Thus,

$$
{ }^{t} \pi \circ{ }^{t} \theta_{\pi}(\varphi \otimes \psi)\left|I={ }^{t} \pi_{1}(\boldsymbol{\varphi}) \otimes{ }^{t} \pi_{2}(\psi)\right| I \neq 0 \text { and }{ }^{t} \pi_{1}(\varphi) \otimes{ }^{t} \pi_{2}(\psi) \mid J=0 .
$$

This completes the proof. 
In the case that both $A$ and $B$ are separable $C^{*}$-algebras the situation is described also in the following manner. We shall show its outline.

Let $\Omega(A), \Omega(B)$ and $\Omega(A \underset{\alpha}{\otimes} B)$ be structure spaces of $A, B$ and $A \underset{\alpha}{\otimes} B$ i.e. spaces of all primitive ideals with hull kernel topology. We shall define the mapping $\Phi$ from $\Omega(A \otimes B)$ to the product space $\Omega(A) \times \Omega(B)$. Let $P$ be a primitive ideal in $A \otimes \otimes_{\alpha}^{\alpha}$ and take an irreducible representation $\pi$ of $A \otimes B$ such as $\pi^{-1}(0)=P$. Its restrictions $\pi_{1}$ and $\pi_{2}$ are factor representations and both $C *$-algebras $\pi_{1}(A)$ and $\pi_{2}(B)$ have no ideal divisors. Hence $\pi_{1}^{-1}(0) \in \Omega(A)$ and $\pi_{2}{ }^{-1}(0) \in \Omega(B)$. Both ideals $\pi_{1}^{-1}(0)$ and $\pi_{2}{ }^{-1}(0)$ do not depend on the choice of the representation $\pi$ and we get the mapping $\Phi$ defined as $\Phi(P)=\left(\pi_{1}{ }^{-1}(0)\right.$, $\left.\pi_{2}{ }^{-1}(0)\right) . \Phi$ is a continuous onto mapping from $\Omega(A \otimes B)$ to $\Omega(A) \times \Omega(B)$. On the other hand, let $P$ and $Q$ be primitive ideals in $A^{\alpha}$ and $B$ and consider the irreducible representations $\pi_{1}$ and $\pi_{2}$ such as $\pi_{1}^{-1}(0)=P$ and $\pi_{2}{ }^{-1}(0)=Q$. The representation $\pi_{1} \otimes \pi_{2}$ is airreducible and $\left(\pi_{1} \otimes \pi_{2}\right)^{-1}(0) \in \Omega(A \otimes B)$ and again the kernel $\left(\pi_{1} \otimes \pi_{2}\right)^{-1}(0)$ does not depend on the choice of $\pi_{1}$ and $\pi_{2}$, so that we can define the mapping $\Psi$ from $\Omega(A) \times \Omega(B)$ into $\Omega(A \otimes B)$ by $\Psi(P, Q)=\left(\pi_{1} \otimes \pi_{2}\right)^{-1}(0)$. The mapping $\Psi$ is also continuous.

Now the composed mapping $\Phi \cdot \Psi$ is the identity map in $\Omega(A) \times \Omega(B)$. While, "the composed mapping $\Psi \circ \Phi$ is the identity map in $\Omega(A \otimes B)$ if and only if $A \otimes_{\alpha} B$ satisfies the condition $(F) . "$ In this case, $\Omega(A \otimes B)$ is homomorphic with the product space $\Omega(A) \times \Omega(B)$.

The relation between the condition $(F)$ and the property $(T)$ is the following; if the homomorphic images of a $C^{*}$-algebra $A$ having the property $(T)$ have always the property $(T)$, then $A \otimes B$ satisfies the condition $(F)$, by 3 of Theorem 5 , for any $C^{*}$-algebra $B$. However it is not known whether the homomorphic images of a $C^{*}$-algbera having the property $(T)$ have also the property $(T)$ or not.

We note at last that if $A \otimes B$ satisfies the condition $(F)$ the equality $K_{1} \otimes K_{2}=K$ holds where $K_{1}, K_{2}$ and $K$ are the largest $G C R$ ideals in $A, B$ and $A \otimes_{\alpha} B$ used in Theorem 4. In fact, if a pure state $\phi \otimes \psi(\varphi \in P(A), \psi \in P(B))$ vanishes on $K_{1} \otimes_{\alpha} K_{2}$ we have $\varphi \mid K_{1}=0$ or $\psi \mid K_{2}=0$, and

$$
<x, \boldsymbol{\varphi} \otimes\rangle=<R_{\varphi}(x), \psi>=<L_{\psi}(x), \varphi>=0
$$

for every $x \in K$ because $R_{\varphi}(K) \subset K_{2}$ and $L_{\psi}(K) \subset K_{1}$ by Theorem 3. Since $K_{1} \underset{\alpha}{\otimes} K_{2} \subset K$, this implies the conclusion.

\section{REFERENCES}

[1] J. Dixmier, Les algèbres d'opérateurs dans l'espace hilbertien, Paris. 1957. 
[2] J. Dixmier, Sur les $C^{*}$-algèbres, Bull. Soc. Math. France, 88(1960), 95-112.

[3] J. Dixmier, Point séparés dans le spectre d'une $C^{*}$-algèbre, Acta Sc. Math., 22(1961), $115-128$.

[4] J. DixmIER, Traces sur les $C^{*}$-algèbres, Ann. Inst. Fourier, 13(1963), 219-262.

[5] J. Glimm, A Stone-Weierstrass theorem for $C^{*}$-algebras, Ann. Math., 72(1960), 216-244.

[6] J. Glimm, Type I $C^{*}$-algebras, Ann. Math., 73(1961), 572-612.

[7] A. GuICHARDET, Caractères et représentations de produits tensoriels de $C^{*}$-algèbres, Ann. Ecole Norm. Sup., 81(1964), 189-206.

[8] A. Guichardet, Sur les produits tensoriels de $C^{*}$-algèbres, Doklady Akad. Nauk 160 (1965), 986-989. (Russian)

[9] I. KAPLANSKY, The structure of certain operator algebra, Trans. Amer. Soc., 70(1951), $139-146$.

[10] N. MochIZUKI, The tensor product of function algebras, Tôhoku Math. Journ., 17(1965), $139-146$.

[11] T. OKAYASU, On the tensor products of $C^{*}$-algebras, Tôhoku Math. Journ., 18(1966), 325-331.

[12] S. SaKAI, On a characterization of type I $C^{*}$-algebras, Bull. Amer. Math. Soc., 72 (1966), 508-512.

[13] R. ShatTen, Theory of cross-spaces, Princeton, 1950.

[14] M. TAKESAKI, On the cross-norm of the direct product of $C^{*}$-algebras, Tôhoku Math. Journ., 16(1964), 111-119.

[15] J. Tомгуама, Tensor products of commutative Banach algebras, Tôhoku Math. Journ., 12(1960), $147-154$.

[16] T. TURUMARU, On the direct product of operator algebras, Tôhoku Math. Journ., 4 (1952), 242-251.

[17] T. Turumaru, On the direct product of operator algebras II, Tôhoku Math. Journ., 5(1953), 1-7.

[18] A. Wulfsohn, Produits tensoriels de $C^{*}$-algèbres, Bull. Sci. Math., 87(1963), 13-27

[19] A. Wulfsohn, Le produit tensoriel de certaines $C^{*}$-algèbres, C.R. Acad. Sc. Paris 258 (1964), 6052-6054.

[20] Z. TAKEDA, Inductive limit and infinite direct product of operator algebras, Tôhoku Math. Journ., 7(1955), 67-86.

FACULTY OF LITERATURE AND SCIENCE

YAMAGATA UNIVERSITY,

YAMAGATA, JAPAN 